\title{
The Tenability of Vibration Parameters of a Sandwich Beam Featuring Controllable Core: Experimental Investigation
}

\author{
Shreedhar Kolekar, ${ }^{1,2}$ Krishna Venkatesh, ${ }^{3}$ Jeong-Seok Oh, ${ }^{4}$ and Seung-Bok Choi ${ }^{5}$ \\ ${ }^{1}$ Mechanical Engineering Department, Jain University, Bengaluru, Karnataka State, India \\ ${ }^{2}$ Mechanical Engineering Department, Satara College of Engineering \& Management Limb, Satara, Maharashtra State 415015, India \\ ${ }^{3}$ Centre for Incubation, Innovation, Research \& Consultancy, Bengaluru, Karnataka State, India \\ ${ }^{4}$ Division of Automotive \& Mechanical Engineering, Kongju National University, Cheonan-si, Chungnam 31080, Republic of Korea \\ ${ }^{5}$ Department of Mechanical Engineering, Inha University, No. 253, Yonghyun-dong, Nam-gu, Incheon 402-751, Republic of Korea
}

Correspondence should be addressed to Seung-Bok Choi; seungbok@inha.ac.kr

Received 23 June 2017; Revised 18 August 2017; Accepted 24 September 2017; Published 19 October 2017

Academic Editor: Kim M. Liew

Copyright (c) 2017 Shreedhar Kolekar et al. This is an open access article distributed under the Creative Commons Attribution License, which permits unrestricted use, distribution, and reproduction in any medium, provided the original work is properly cited.

\begin{abstract}
This study presents experimental results of the vibration parameters of a sandwich beam featuring magnetorheological (MR) fluid as core material. For simplicity, the sandwich beam is considered as a single-degree-of-freedom (SDOF) system and the governing equation is derived in time and frequency domains. Then, from the governing equation, the vibration parameters which can be controllable by external stimuli are defined or obtained. These are the field-dependent natural frequency, damping factor, loss factor, and quality factor of the sandwich beam. Subsequently, a sandwich beam incorporating with controllable MR fluid core is fabricated and tested to evaluate the vibration parameters. MR fluid is prepared using the engine oil, iron particles, and grease as an additive and it is filled into the void zone (core) of the sandwich beam. The fabricated beam is then tested at four different conditions and the vibration parameters are numerically identified at each test. It is shown that both the natural frequency and damping property can be tuned by controlling the intensity of the magnetic field applied to MR fluid domain.
\end{abstract}

\section{Introduction}

The development of sandwich structural systems with integrated control capabilities of modal characteristics is crucial to control unwanted vibrations and to avoid resonance problem due to external disturbances. These systems can provide higher flexural stiffness to weight ratio, lower lateral deformations, higher buckling resistance, and higher the natural frequencies. The distributed control force throughout the sandwich structures could be achieved by embedding controllable smart materials as cores or layers between two base structures. This approach can facilitate structure vibration control over a broad range of frequencies through variations in distributed stiffness and damping properties in response to applied stimuli. These structures are called smart sandwich structures like a smart structure in which both the natural frequency and damping property can be controlled by applying external fields such as voltage and current. The development of electrorheological (ER) fluid based sandwich structures was initiated by Gandhi et al. [1-3]. In this work, it has been shown that the dynamic characteristics of ER fluid based sandwich structures can be tuned showing the increment of damping ratio and natural frequencies as the electric field increases. As extension works, Choi et al. showed that the transient vibration of a flexible link robot could be effectively controlled by applying control voltage and also demonstrated that mode shapes of sandwich plate with ER fluid core could be controlled by localizing core zones [4-7]. Experiments were also performed using various ER fluid cores including corn starch, corn oil, and zeolitesilicone oil. Substantial variations in natural frequencies of sandwich beams with these cores were observed by changing the applied electric field $[8,9]$. Leng et al. [10] experimentally investigated the vibration analysis of ER fluid composite sandwich beam. It was concluded that the first three modes of natural frequencies and damping factors were increased with 
increasing the applied electric field. Yalcintas and Coulter [11] developed an analytical model to characterize the forced vibration response of a simply supported ER sandwich beam using RKU (Ross-Kervin-Ungar) model. The numerical solutions were validated through experimental measurements. Yeh and Chen $[12,13]$ evaluated the variation in the stiffness and natural frequency of the sandwich plate with ER fluid by varying the applied electric field. They concluded that the resonance frequencies of the sandwich plate could be increased with increase in electric field and decreased with increase in thickness of the ER fluid core. It was also found that the thickness of the core has a significant effect on the stability of the sandwich structure system.

Since magnetorheological (MR) fluid has same characteristics as ER fluid except external stimuli, several researchers have attempted to develop sandwich structures featuring MR fluid cores. Yalcintas and Dai $[14,15]$ investigated dynamic responses of MR fluid adaptive sandwich beam using the energy approach and compared the responses with the structure employing ER fluid. It was concluded that the natural frequencies of MR fluid based adaptive sandwich beam could be nearly twice those of ER fluid based sandwich beam. Sun et al. [16] analytically studied the dynamic responses of a MR fluid sandwich beam using the energy approach and the results are validated by experimental measured data. Oscillatory rheometry techniques were used to carry out experiments to develop the relationship between the applied magnetic field and complex shear modulus of the MR fluid. Yeh and Shih [17] studied theoretically the dynamic responses of MR material based adaptive beam under axial harmonic load using DiTaranto sandwich beam theory. $\mathrm{Hu}$ et al. [18] investigated the vibration characteristics of MR fluid based sandwich beam using DiTaranto sixth-order partial differential equation. It was shown that the natural frequencies and loss factors of the MRF beam were increased with increasing applied magnetic field strength. Vasudevan et al. [19] derived the governing differential equations of motion by FEM and Ritz formulations for a sandwich beam with MR fluid treatment and validated through experiments conducted on a cantilever sandwich beam. Various parametric studies were performed in terms of variations of the natural frequencies and loss factor as functions of the applied magnetic field and thickness of the MR fluid layer for various boundary conditions. Lara-Prieto et al. [20] experimentally investigated the controllability of vibration characteristics of MR fluid based sandwich beams under various magnetic field intensities. The effects of applied magnetic field at partial and full length of MR fluid sandwich beam were analyzed. The effectiveness of the linear quadratic regulator and flexible mode shape method based optimal control techniques on controlling transient and forced vibration responses of a fully and partially treated MR fluid sandwich were investigated by Vasudevan et al. [21]. The vibration response of a MR fluid sandwich plate was analyzed by Li et al. [22]. It was shown that the natural frequencies increase with increase in applied magnetic field. However, the loss factors decrease in higher modes with increase in magnetic field. Yeh [23] studied the free vibration characteristics of a magnetorheological elastomers based sandwich plate. The loss factor and the natural frequencies of the sandwich plate were evaluated under various magnetic fields. Rajamohan et al. [24] studied to find the properties and also vibration response of a partially treated multilayer MR fluid beam and governing equations have been derived for partially treated multilayer prototype beam using finite element and Ritz method and compared the results with experimental and Ritz method; the effects of length and locations of MR fluid layers on the properties of the beam are investigated under different magnetic field conditions and demonstrated upon the boundary conditions and mode of vibration to be controlled for the effective vibration suppression has been derived. Rajamohan et al. [25] investigated governing equations for a partially treated MR fluid layer using FEM and Ritz approach, two different configurations of a partially treated MRF sandwich beam are considered, and the parametric studies were performed to investigate the influence of intensity of an external magnetic field and location and length of MR fluid layers on the dynamic characteristics of the structure with different boundary conditions. Rajamohan et al. [26] worked on governing equations for nonhomogeneous multilayer MR beam which were derived under nonhomogeneous conditions using FEM and Ritz formulation; the beam is formed using three different types of MR fluid and has various shear modulus properties and results showed that natural frequency at higher modes could be controlled by locating the MR fluid layers at desired locations. The natural frequency at higher modes could be increased with decreasing the length of MR fluid layer and it confirms that amplitude of vibration could be easily reduced using controllable MR fluid having different shear modulus located at the desired location and applied to more critical parts to realize more efficient vibration control. Rajamohan and Natarajan [27] worked on the dynamic behaviour of a rotating MRF sandwich beam using FEM and Ritz approach; various parametric studies were performed to study the effect of magnetic field on natural frequency and loss factors. The effect of thickness of MR fluid on natural frequency and effect of rotational speed and hub radius on natural frequencies corresponding to all the modes of vibration of rotating MR sandwich beam increased significantly with the increase in applied magnetic field intensity. Momeni et al. [28] investigated MRF sandwich beam using both experimental and simulation processes. FEM model is used to simulate vibration response under random loading and FEM approach is validated with experimental one and shows that as the magnetic field increases correspondingly the natural frequency for the sandwich beam increases. Walikar et al. [29] worked on engine oil based MR fluid using nickel as magnetisable particle and oleic acid as a surfactant with variation in concentration of nickel particles and found that effect of different magnetic field and concentration of magnetisable particles increase the natural frequency of the beam and amplitude of vibration decreases. Joshi [30] worked on vibration control of cantilever sandwich beam using laboratory prepared MR fluid and observed the variations in vibration amplitude and shifts in magnitude of resonance natural frequency. So the variations usually decreases in vibration amplitude and loss factors and increase in natural frequency as electric/magnetic field increases. However the 
variations in above parameters were more effective in $M R$ adaptive structures compared with ERF structures.

Despite many research works on sandwich structures having controllable cores such as ER and MR fluids, the study on the vibration parameters which characterize vibration motions of sandwich structures is considerably rare. It is noted that in order to define or explain the vibration parameters such as loss factor a specific and simple model which governs vibration motions needs to be adopted. Many of previous works on smart sandwich structures provide the vibration parameters which are directly obtained from experimental tests without the specific definition. Consequently, this work presents criteria to evaluate the vibration parameters of smart sandwich beams considering a single-degree-of freedom (SDOF). After defining the vibration parameters from the governing equation of the SDOF, a sandwich beam with controllable MR fluid as core was fabricated and tested under free and forced vibration conditions at different magnetic fields. Then, the field-dependent vibration parameters such as natural frequency and loss factor are obtained and compared at four different conditions: empty sandwich beam, MR fluid sandwich beam at $0 \mathrm{~T}, \mathrm{MR}$ fluid sandwich beam at $0.1 \mathrm{~T}$, and MR fluid sandwich beam at $0.2 \mathrm{~T}$, respectively. It is shown that the vibration parameters heavily depend on the magnetic intensity.

\section{Vibration Parameters of SDOF Model}

As mentioned in Introduction, several advantages can be achieved by applying smart sandwich structures due to the controllability of core materials. Some of advantages are as follows.

(i) Control of Vibration Amplitude at Resonance. Damping can be used to control the excessive resonance vibrations which may cause high stresses leading to the permanent failure. It should be used in conjunction with other appropriate measures to achieve the most satisfactory approach for random excitations and it is not possible to detune the system and design to keep random stresses with acceptable limit without ensuring that the damping in each mode at least exceeds a minimum specified value. This is a case for sonic fatigue of aircraft fuselage, wing, and control surface panels when they are excited by the jet noise and boundary layer turbulence induced excitations.

(ii) Noise Control. Damping is useful for the control of noise radiation from vibrating surfaces or control of noise transmission through a vibrating surface.

(iii) Damping Phenomenon. The damping is nothing but the energy dissipation in a vibrating structures. The energy which is dissipated in vibrating structures usually depends upon physical mechanisms that exist in the active structures and the physical mechanisms are very complicated physical processes and it is very difficult to analyze the system. The type of damping phenomenon that existed in the structures and usually depends upon the mechanism, which predominates under the given situation, is very essential. In a true physical

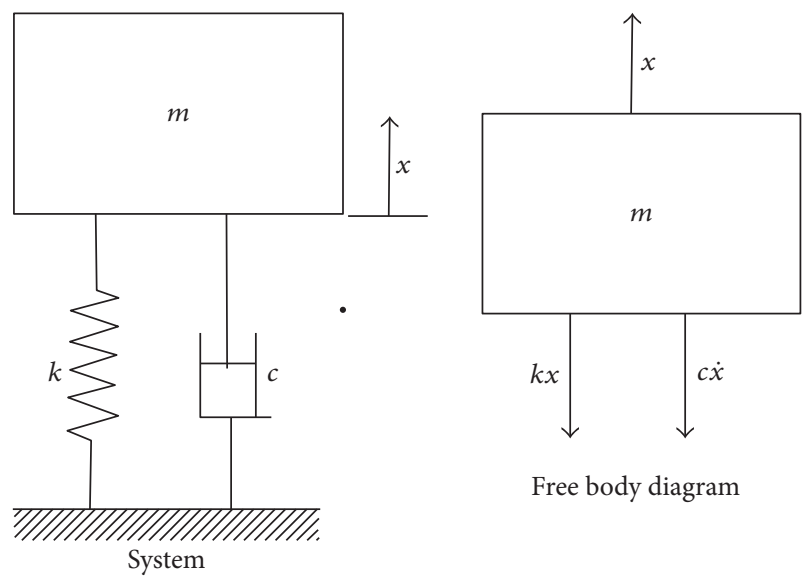

FiguRE 1: Single degree-of-freedom system.

situation, the development of a mathematical equation of motion for the vibrating structure with a physical damping mechanisms is very significant. In the year 1970, Scanlan [31] has found the mathematical damping model which does not give much information.

In order to define vibration parameters of smart sandwich structures featuring controllable core materials, the SODF shown in Figure 1 is adopted because spring-mass-damper model is an oversimplification of the most real structures. In a free vibration system under the undamped case, the vibration response of the SDOF system will never die out. The easiest approach to introduce a dissipation will take place in viscous dashpot system as shown in the figure. The damping force $\left(F_{d}\right)$ which is directly proportional to instantaneous velocity is given by

$$
F_{d}=c \dot{x}
$$

where $c$ is called a dashpot or viscous damping constant. The loss factor $(\eta)$ which measures damping phenomenon and is defined as the sinusoidal excitation of the system to the corresponding sinusoidal response of the system is as follows:

$$
\eta=\frac{c|\omega|}{k}
$$

where $k$ is the stiffness of the system. The above equation is similar to the equation for the viscoelastic systems developed by the Ungar and Kerwin's [32]. Eq. (2) shows a linear dependence between loss factor to driving frequency and inversely proportional to the stiffness of the system. This kind of frequency dependence has been discussed by Crandall in the year 1970 [33], but in actual practices it is not possible this form and in such a case often resorts to an equivalent ideal dashpot system. The theoretical objections to the approximately constant value of damping over a range of frequency, as can be observed in aeroelasticity problems, have been raised by Naylor in the year 1970 [33]. From (2), the frequency- dependent dashpot system is given by

$$
c|\omega|=\frac{k \eta(\omega)}{|\omega|} .
$$


From Figure 1, the frequency domain representation of equation of motion can be written as follows:

$$
\left[-m \omega^{2}+i \omega c(\omega)+k\right] X(i \omega)=F(i \omega),
$$

where $X(i \omega)$ is the response function and $F(i \omega)$ is the excitation function. The viscous damping or dashpot has frequency dependence. Substituting (3) into (4) yields the following:

$$
\left[-m \omega^{2}+k\{1+i \eta(\omega) \operatorname{sgn}(\omega)\}\right] X(i \omega)=F(i \omega),
$$

where $\operatorname{sgn}(\omega)$ is the signum function. For the "time domain" representations of (3) and (4) are expressed as follows:

$$
m \ddot{x}+c(\omega) \dot{x}+k x=f .
$$

Then, by assuming the response function as $x(t)=$ $x_{0} \cos (\omega t-\delta)$ for the harmonic function at the frequency $(\omega)$ and also the phase $\operatorname{lag}(\delta)$, the relationship between forcing function to the excitation can be related by

$$
F=k x+\frac{k \eta}{|\omega|} \frac{d x}{d t},
$$

where $k$ is the stiffness. $\eta=\tan \delta$ is called the loss factor for inertial and stiffness properties. The phase angle $\delta$ lies between $0^{\circ}$ and $90^{\circ}$, and the loss factor $\eta$ lies between 0 and $\infty$. The relationship exists between $\eta$ and $\delta$ values.

On the other hand, the governing equation of the system shown in Figure 1 is given by

$$
m \frac{d^{2} x}{d t^{2}}+k x+\frac{k \eta}{\omega} \frac{d x}{d t}=F_{0} \cos \omega t .
$$

For the harmonic response function in a steady state condition, transients at any start have usually died out. If the stiffness $(k)$ and loss factor $(\eta)$ depend upon frequency for real materials, then the maximum amplitude at the resonance frequency $\left(\omega_{r}\right)$ can be written as $\omega_{r}=\sqrt{ } k / m$. Amplification factor $(A)$ is nearly equal to $1 / \eta(\omega r)$ and the loss factor is given by

$$
\eta=\frac{1}{A}=\frac{\Delta \omega}{\omega_{r}}
$$

where $\Delta \omega$ is the separation of the frequencies; its response is $1 / \sqrt{ } 2$ times the peak response. In summary, $\eta=1 / A=$ $\Delta \omega / \omega_{r} ; \Delta \omega$ is the frequencies separation response which is equivalent to $(1 / \sqrt{ } 2) X$. The peak response which is known as half power bandwidth is equal to $\eta=1 / A=\Delta \omega / \omega_{r}$.

Now, the following evaluation (vibration) parameters are defined as criteria for vibration characteristics of smart sandwich structures.

Natural Frequency or Velocity Resonant Frequency $\left(\omega_{n}\right)$. The calculation of natural frequencies is of major importance in the study of vibrations. Because of friction and other resistances vibrating systems are subjected to damping to some degree due to dissipation of energy. If the damping is small, it has very little effect on natural frequency of the system.

$$
\begin{aligned}
& \text { (i) Natural frequency }\left(\omega_{n}\right)=\frac{2 \pi}{T} \mathrm{rad} / \mathrm{sec} \\
& \text { (ii) Time period }(T)=\frac{1}{\omega} \mathrm{sec} \text {. }
\end{aligned}
$$

(iii) Damping Factor ( $\zeta$ ). The half power point method is another method for finding the damping factor form graphical representation and it provides fairly accurate results. Suppose that the natural frequency $\left(f_{n}\right)$ can be measured at the peak amplitude $\left(X_{\max }\right)$, and $f_{1}$ and $f_{2}$ are two values of natural frequency of the unit of $\mathrm{Hz}$. Then, the damping factor can be obtained by

$$
\zeta=\frac{f_{2}-f_{1}}{2 f_{n}} .
$$

(iv) Peak Amplitude. The maximum displacement of a vibrating body, in vibration study amplitude, should be minimized and natural frequency is to be increased.

(v) Logarithmic Decrement $(\Delta)$. Whenever the damping system is stroked by the impulse force, the response is exponentially decreased and from this the logarithmic decrement is obtained by

$$
\Delta=\ln \frac{x_{1}}{x_{2}}=\ln \frac{x_{n}}{x_{n+1}} .
$$

It is noted that the above definition is only useful for viscous and hysteretic type of damping within limits because the ratios are equal for the viscous cycles. On the other hand, using forcing function and excitation function expressed in Fourier transform the solution of (4) can be expressed as follows:

$$
x(t)=\frac{1}{2 \pi} \int_{-\infty}^{\infty} \frac{F e^{j \omega t} d \omega}{k}-m \omega 2+j k \eta .
$$

The above equation consists of two parts; one is real and another is an imaginary function, and then the following solution is obtained:

$$
x(t)=\frac{F}{\pi} \int_{0}^{\infty} \frac{\left(k-m \omega^{2}\right)(\cos \omega t)+k \eta \sin \omega t}{\left(k-m \omega^{2}\right)^{2}+(k \eta)^{2}} d \omega .
$$

The values of stiffness $(k)$ and loss factor $(\eta)$ are not to be constants for the real systems in a certain wide frequency range. For small values of loss factor $(\eta)$, the accuracy is the best and the following equations are obtained:

$$
\begin{aligned}
x(t) & =\frac{F}{\sqrt{k m}} e^{-1 / 2 \eta t \sqrt{k} / m} \sin t \sqrt{\frac{k}{m}} . \\
\Delta & =\frac{\pi \eta}{2}
\end{aligned}
$$

(vi) Resonance Frequencies. The peak values of the displacement, velocity, and acceleration response of a system 


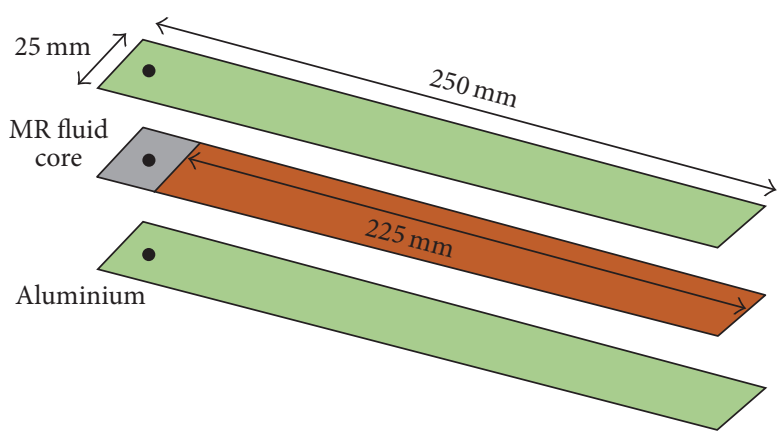

(a)

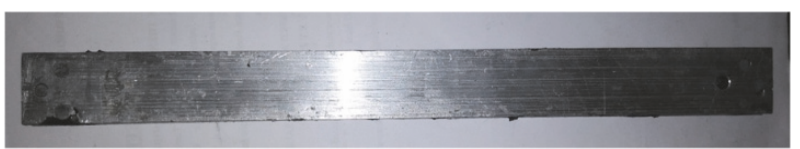

(b)

FIgURE 2: Fabricated sandwich beam with MR fluid core; (a) schematic, (b) photograph.

undergoing forced, steady state vibration occur at slightly different forcing frequencies. Since a resonance frequency is defined as the frequency for which the response is maximum, a simple system has three resonance frequencies if defined only generally. The natural frequency is different from any of the resonance frequencies. There is a relationship between damped natural frequency and undamped natural frequency using the damping ratio.

Now, the following vibration parameters to evaluate smart sandwich structures are achieved as follows:

(i) Damped Frequency: $\omega_{d}=\omega_{n} \sqrt{ } 1-\zeta^{2}$

(ii) Displacement Resonant Frequency:

$$
D f=\omega_{n} \sqrt{ } 1-2 \zeta^{2}
$$

(iii) Acceleration Resonant Frequency:

$$
A f=\frac{\omega_{n}}{\sqrt{ } 1}-2 \zeta^{2}
$$

(iv) Loss Factor: $\eta=2 \zeta$

(v) Quality Factor $=\frac{1}{2} \zeta$

(vi) Specific damping capacity: $S c=4 \pi \zeta$.

\section{Design and Manufacture of Sandwich Beam}

A simple sandwich beam is selected which is capable of withstanding load primarily by resisting bending. Beams are traditionally descriptions of building or civil engineering structural elements, but smaller structures such as truck or automobile frames, machine frames, and other mechanical and structural systems contain beam structure that are designed and analyzed in a similar fashion. So this simple mechanical model can be implemented in to more complex structures. Here in this research work the controllable capabilities of MR fluid in adaptive structures were analyzed in real time. In order to evaluate the criteria for vibration characteristics of sandwich structures, a sandwich beam with MR fluid core was fabricated and tested in this work. Figure 2 presents the schematic diagram and photograph
TABLE 1: Dimensions of each layer.

\begin{tabular}{lcc}
\hline SI number & Type of layer & Dimensions of layer \\
\hline$(01)$ & Base structure & $250 \times 25 \times 3 \mathrm{~mm}$ \\
$(02)$ & Core layer $\left(\mathrm{h}_{2}\right)$ & $225 \times 20 \times 3 \mathrm{~mm}$ \\
\hline
\end{tabular}

TABLE 2: Properties of beam and MR fluid.

\begin{tabular}{lcc}
\hline SI number & Type of layer & Properties \\
\hline$(01)$ & $\begin{array}{c}\text { Base structure } \\
\text { (aluminium) }\end{array}$ & $\begin{array}{c}\text { Density }=2700 \mathrm{~kg} / \mathrm{m}^{3} \\
\text { Young's modulus }=70 \mathrm{Gpa}\end{array}$ \\
$\begin{array}{c}\text { Core Layer } \\
(\text { MR fluid) }\end{array}$ & Density $=2781 \mathrm{~kg} / \mathrm{m}^{3}$ \\
\hline
\end{tabular}

of the proposed smart sandwich beam tested in this work. MR fluid sandwich beam consists of constrained layer, base structures, and MR fluid core between upper and lower base structures which are made of aluminium. The dimensions of each layer are given in Table 1 and properties of the sandwich beam with MR fluid core are given in Table 2. The beam top and bottom base layer has length of $250 \mathrm{~mm}$ and width of $25 \mathrm{~mm}$, while MR fluid core length is $200 \mathrm{~mm}$ with the width of $20 \mathrm{~mm}$. The thickness of each aluminium layer and core layer is $3 \mathrm{~mm}$. At the clamped end of the plate a small $45 \mathrm{~mm}$ extra plate is fixed in between two aluminium strips; other surfaces of the beam are sealed with silicon rubber; for filling up of MR fluid in a cavity a small hole of $5 \mathrm{~mm}$ size diameter was drilled in each side of the beam. From one end of the of the beam MR fluid is injected using hypodermic syringe; this allowed air bubbles to escape from other side of the hole and finally two holes were sealed and allowed to dry it.

3.1. Synthesis of MR Fluid. MR fluid used in this work was made according to the following steps. Step 1. Pour the desired amount of carrier oil into the beaker. Stir it for one hour using mechanical stirrer. Step 2. Take a small quantity of grease and mix it with carrier oil and stir it in a mechanical stirrer for 2-3 hours until the grease particles will be completely dissolved and suspended in carrier oil. After complete mixing slowly pour the iron particles in mixture and again stir it for 6-8 hours. Step 3. Prepared MR fluid 


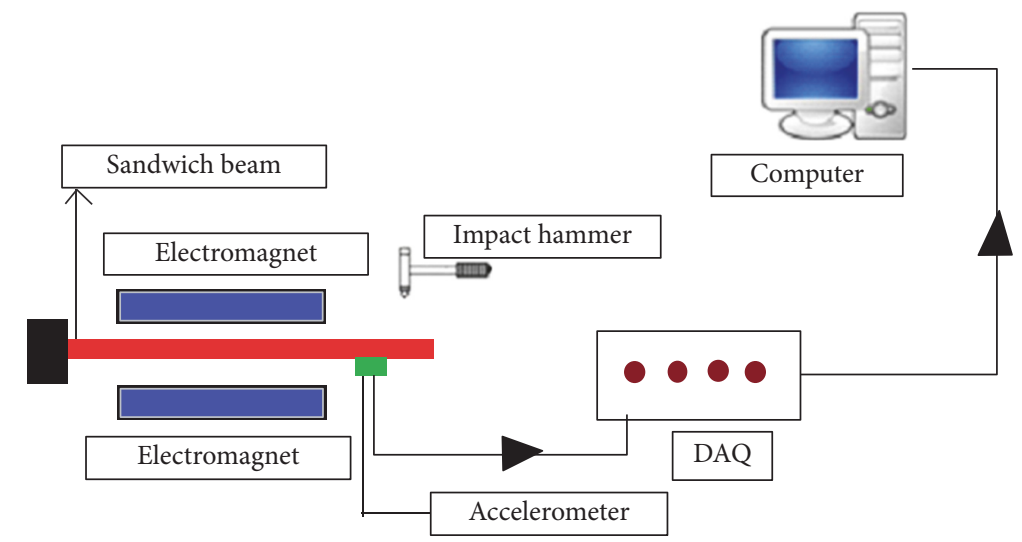

FIGURE 3: Schematic Experimental test rig for the MRF sandwich beam.

TABle 3: Properties of SAE grade 20W-40.

\begin{tabular}{lcc}
\hline $\begin{array}{l}\text { SI } \\
\text { number }\end{array}$ & Properties & Values \\
\hline$(01)$ & Density at $20^{\circ} \mathrm{C}$ & Density $=876 \mathrm{~kg} / \mathrm{m}^{3}$ \\
$(02)$ & Kinematic viscosity at $100^{\circ} \mathrm{C} \mathrm{cSt}$ & $13.5-15.5$ \\
$(03)$ & Viscosity index & 110 \\
$(04)$ & Flash point & $200^{\circ} \mathrm{C}$ \\
\hline
\end{tabular}

is ready for the use. In this research work low viscosity silicon oil is used for the preparation of MR fluid in the laboratory. The composition is summarized as follows: carrier oil-Engine oil (SAE Grade 20W-40) of 70\% and Magnetic particles-Electrolytic Iron powder (Industrial Metal Powders Pune India) of $30 \%$ Additives, Commercial Grease of $10 \%$. The sample is denoted as SAE Grade 20W-40 (Mobil Diesel Special Multi Grade Diesel Engine oil) and the principal properties are given in Table 3.

3.2. Experimental Setup. The main elements which were employed in this work include MR fluid sandwich beam, accelerometer, exciter, power amplifier, Data acquisition system (DAQ), Electromagnets, Display unit, and Impulse hammer. (i) Accelerometer. Place the accelerometer on the free end of the beam by applying gum solution on it and connect it to the data acquisition system (DAQ). It senses the analogous displacement of the beam and sends it to the data acquisition system (DAQ). Here in this research work Kistler model 9722A2000, an accelerometer which has the sensitivity of $10.84 \mathrm{mV} / \mathrm{g}$ is used. (ii) Exciter. It is used to give desired excitation to the beam; the power is given to the exciter by controller which is connected with the computer to select the excitation parameter. The different types of excitation can be generated by the exciter by using power amplifier Ex: Sine swept sine, rectangular, triangular, and so on. In case of forced vibration we can use swept sine signal in which user has to select the initial and final frequency and swept rate. Here in this research work LDSV101-permanent magnet shaker is used in this experiment (sine peak force $8.9 \mathrm{~N}$ ).
It is extensively used in industries and it is suitable for the analysis of dynamic behaviour of the structures and materials (iii) Power Amplifier. It is connected to the exciter to give particular excitation frequency to the exciter. Here in this research work TECHRON 5507 is used as power amplifier. The high level of self-excitation is achieved by increasing the gain of the input signal and applied to the exciter. (iv) Data Acquisition System. It takes vibration signal from accelerometer and encodes in digital form. It receives the voltage signal from the accelerometer and calibrates the data into equivalent accelerometer scale through its sensitivity and sends it to the computer by using software. These data are analyzed as a time history (displacement-time) and frequency domain (i.e., FFT). Here NIC DAQ 9174 is designed for the small portable and mixed measurement test system. And it consists of high output speed USB communication NI signal streaming technology and also lab view (laboratory Virtual Instrumentation Engineering work bench) logging software. ( $v$ ) Electromagnets. Electromagnets are used to generate magnetic field around the sandwich beam; here magnetic field was applied up to 0.2 T. (vi) Impulse Hammer. This looks like an ordinary hammer. On hitting the impact hammer on cantilever structure an equal and opposite force is sensed by the beam. This generates vibrations in the sandwich beam. (vii) Display Unit. Data acquisition system is connected to the computer and it shows the results.

\subsection{Experiment Procedure}

3.3.1. Free Vibration Study. Free vibrations can be defined as oscillations about a system's equilibrium position that occurs in the absence of an external excitation. (i) Place the sandwich beam to hold in one side and other end is free, fix the accelerometer on the beam by applying the gum solution on it, and connect it to the accelerometer with data acquisition system connected to the computer with the help of USB connector. (ii) Check if the connections are properly arranged or not. (iii) Open the lab view software in computer. (iv) Hammer the beam with the help of hammer under different field conditions. (v) Response curves were obtained. Figure 3 shows a schematic representation of free vibration study. 


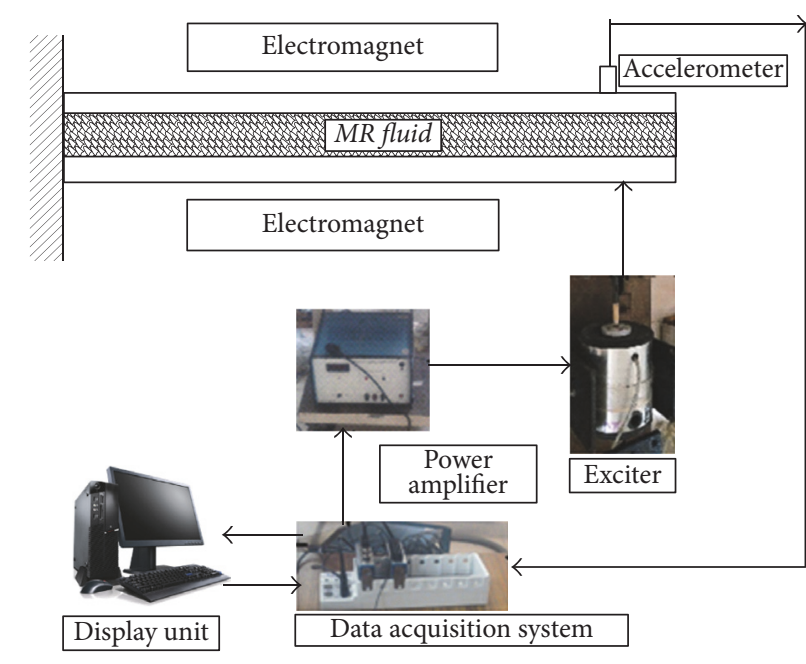

FIGURE 4: Experimental test rig for the MRF sandwich beam.

3.3.2. Forced Vibration Study. The vibration that takes place under the excitation of external forces is called forced vibration. If excitation is harmonic, the system is forced to vibrate at excitation frequency. If the frequency of excitation coincides with one of the natural frequencies of the system, a condition of resonance is encountered and dangerously large oscillations may result, which results in failure of major structures, that is, bridges, buildings, or airplane wings. MR fluid sandwich beam was clamped at the one end and the other end is free, that is, cantilever condition. Figures 4 and 5 show the schematic representation and photograph of an experimental apparatus which integrates with electromagnets, accelerometer, exciter, power amplifier, and signal analysis. The equipment used in the experiment test rig includes lab view programming and data acquisition (DAQ) board which is small and portable. The accelerometer is to sense the displacement of the beam due to the excitation by the magnet shaker. The power amplifier delivers accurate high power levels with complete self-protection for dependable operation. Initially, the empty sandwich beam is fixed to the rig using gum solution and connect in to DAQ board which is connected to computer using USB connector. The power is supplied to the exciter by increasing gain of amplifier whenever the excitation is given to the exciter having frequency range of 20 to $100 \mathrm{~Hz}$. Subsequently, the sandwich beam with MR fluid core is prepared and tested by applying the magnetic field up to $0.2 \mathrm{~T}$. It is noted that the parameters like amplitude and exciting frequency are set to be same for all tests. Experiment Procedures are summarized as follows. (i) Clamp one end of the sandwich beam and other end is free. (ii) Place accelerometer at the free end of the sandwich beam using gum solution to measure vibration response and connect it to the data acquisition system DAQ board system to personnel computer using personal computer. (iii) During setting of swept sine parameter make sure that vibration measurement software of the time domain is greater than the total time of excitation. (iv) Make setting to generate swept sine from exciter to excite sandwich beam. (v) Start the experiment by giving force signal to the exciter using the power amplifier and allow the beam to vibrate. (vi) Record all data obtained from accelerometer in the form of variation response with time by varying by using lab view software. (vii) Repeat the experiment for different conditions.

\section{Experimental Results and Discussions}

The test conditions of the smart sandwich beam are classified as follows: (i) empty beam, (ii) with MR fluid core at magnetic field $B=0$, (iii) with MR fluid core at magnetic field $B=0.1 \mathrm{~T}$, and (iv) with MR fluid core at magnetic field $B=0.2 \mathrm{~T}$. Figure 6 presents the frequency responses of the proposed smart sandwich achieved at three different conditions. It is clearly observed from this figure that both the natural frequency and the peak value are significantly changed by the intensity of the magnetic field. Specifically, the natural frequency in the absence of the magnetic field is identified as $33.665544 \mathrm{~Hz}$ and this is increased up to $40.331989 \mathrm{~Hz}$ by applying the magnetic field of $0.2 \mathrm{~T}$. The vibration parameters discussed in the previous section have been identified in this test and the results are summarized in Table 4 . It is seen that the damping factor of the sandwich beam without the field is evaluated as 0.013719 and it is reduced to 0.009219 by applying the magnetic field of $0.2 \mathrm{~T}$. Table 5 presents the detailed variation of the vibration parameters of four different cases. The results presented in this work clearly indicate that the vibration parameters of smart sandwich beam such as natural frequency and loss factor can be adaptively tuned as a function of the magnetic field. This tuning capability can be extended to more advanced control capability of vibration characteristics in real time. This can provide several benefits in vibration environment of flexible structures such as resonance avoidance.

\section{Conclusion}

In this work, principal criteria for the evaluation of vibration characteristics of sandwich structures with controllable core materials were discussed and investigated by undertaking an experimental work on the sandwich beam with controllable MR fluid core. After adopting the sandwich beam as a SDOF system, the vibration parameters such as natural frequency, damping ratio, loss factor, and quality factor were derived and defined. Subsequently, in order to evaluate the vibration parameters, a sandwich beam featuring MR fluid consisting of engine oil and iron particles was fabricated and tested under four different conditions. It has been identified that the natural frequency of the empty beam is higher than the case with MR fluid core in the absence of the magnetic field. This is due to the increment of the mass of MR fluid mainly attributed by iron particles. It has been also seen that the natural frequency and quality factor of the smart sandwich beam are increased as the field intensity increases, while the loss factor is decreased as the magnetic field increases. This directly means that both the natural frequency and damping property of the smart sandwich beam can be adaptively controlled by integrating an appropriate control strategy. This kind of salient benefit can provide several 




FIGURE 5: Photograph of experimental test rig for the MRF sandwich beam.

TABLE 4: Vibration parameters of sandwich beam with MR fluid core for free vibration system.

(a)

\begin{tabular}{lcccccc}
\hline $\begin{array}{l}\text { SI } \\
\text { number }\end{array}$ & $\begin{array}{c}\text { Magnetic field } \\
\text { condition }\end{array}$ & $\begin{array}{c}\text { Natural } \\
\text { frequency }\left(\omega_{n}\right) \\
(\mathrm{Hz})\end{array}$ & $\begin{array}{c}\text { Time } \\
\text { period } \\
\text { Sec }\end{array}$ & $\begin{array}{c}\text { Peak } \\
\text { amplitude } \\
(\mathrm{mm})\end{array}$ & $\begin{array}{c}\text { Damped frequency } \\
\left(\omega_{d}\right) \\
(\mathrm{Hz})\end{array}$ & $\begin{array}{c}\text { Damping } \\
\text { factor } \\
(\zeta)\end{array}$ \\
\hline$(01)$ & Without MR fluid & 24.99 & 0.2514 & 13.82 & 24.96 & 0.046 \\
$(02)$ & With MR fluid 0T & 25.01 & 0.2512 & 13.21 & 24.98 & 0.038 \\
$(03)$ & $0.1 \mathrm{~T}$ & 25.90 & 0.2425 & 13.19 & 25.88 & 0.037 \\
$(04)$ & $0.2 \mathrm{~T}$ & 25.99 & 0.2417 & 12.92 & 25.97 & 0.035 \\
\hline
\end{tabular}

(b)

\begin{tabular}{lcccccc}
\hline $\begin{array}{l}\text { SI } \\
\text { number }\end{array}$ & $\begin{array}{c}\text { Loss factor } \\
(\eta)\end{array}$ & $\begin{array}{c}\text { Logarithmic } \\
\text { decrement } \\
(\Delta)\end{array}$ & $\begin{array}{c}\text { Quality } \\
\text { factor } \\
(Q)\end{array}$ & $\begin{array}{c}\text { Specific } \\
\text { damping } \\
\text { capacity (Sc) }\end{array}$ & $\begin{array}{c}\text { Displacement } \\
\text { resonant frequency } \\
(\text { Df) }\end{array}$ & $\begin{array}{c}\text { Acceleration } \\
\text { resonant } \\
\text { frequency (Af) }\end{array}$ \\
\hline$(01)$ & 0.092 & 0.2893 & 10.86 & 0.578 & 24.93 & 25.04 \\
$(02)$ & 0.076 & 0.2400 & 13.15 & 0.477 & 24.98 & 25.05 \\
$(03)$ & 0.074 & 0.3231 & 13.51 & 0.464 & 25.86 & 25.93 \\
$(04)$ & 0.070 & 0.2248 & 14.28 & 0.439 & 25.95 & 26.02 \\
\hline
\end{tabular}


TABLE 5: Vibration parameters of sandwich beam with MR fluid core for forced vibration system.

(a)

$\left.\begin{array}{lcccccc}\hline \begin{array}{l}\text { SI } \\ \text { number }\end{array} & \begin{array}{c}\text { Magnetic } \\ \text { field } \\ \text { condition }\end{array} & \begin{array}{c}\text { Natural } \\ \text { frequency }\left(\omega_{n}\right) \\ (\mathrm{Hz})\end{array} & \begin{array}{c}\text { Time } \\ \text { period } \\ \text { Sec }\end{array} & \begin{array}{c}\text { Peak } \\ \text { amplitude } \\ (\mathrm{mm})\end{array} & \begin{array}{c}\text { Damped frequency } \\ \left(\omega_{d}\right)\end{array} & \begin{array}{c}\text { Damping } \\ (\mathrm{Hz})\end{array} \\ \hline(01) & \begin{array}{c}\text { Without MR } \\ \text { fluid } \\ \text { With MR }\end{array} & 32.99 & 0.19 & 4.50744 & 32.99 \\ (\zeta)\end{array}\right)$

(b)

\begin{tabular}{lcccccc}
\hline $\begin{array}{l}\text { SI } \\
\text { number }\end{array}$ & $\begin{array}{c}\text { Loss factor } \\
(\eta)\end{array}$ & $\begin{array}{c}\text { Logarithmic } \\
\text { decrement } \\
(\Delta)\end{array}$ & $\begin{array}{c}\text { Quality factor } \\
(Q)\end{array}$ & $\begin{array}{c}\text { Specific } \\
\text { damping } \\
\text { capacity }(\text { Sc) }\end{array}$ & $\begin{array}{c}\text { Displacement } \\
\text { resonant frequency } \\
(D f)\end{array}$ & $\begin{array}{c}\text { Acceleration } \\
\text { resonant } \\
\text { frequency (Af) }\end{array}$ \\
\hline$(01)$ & 0.029174 & 0.045826412 & 34.27 & 0.1833 & 32.99 & 33.00 \\
$(02)$ & 0.027438 & 0.043099509 & 36.44 & 0.1723 & 33.66 & 33.67 \\
$(03)$ & 0.020638 & 0.032418094 & 48.45 & 0.1296 & 34.99 & 35.00 \\
$(04)$ & 0.018438 & 0.028962342 & 54.23 & 0.1158 & 40.33 & 40.33 \\
\hline
\end{tabular}

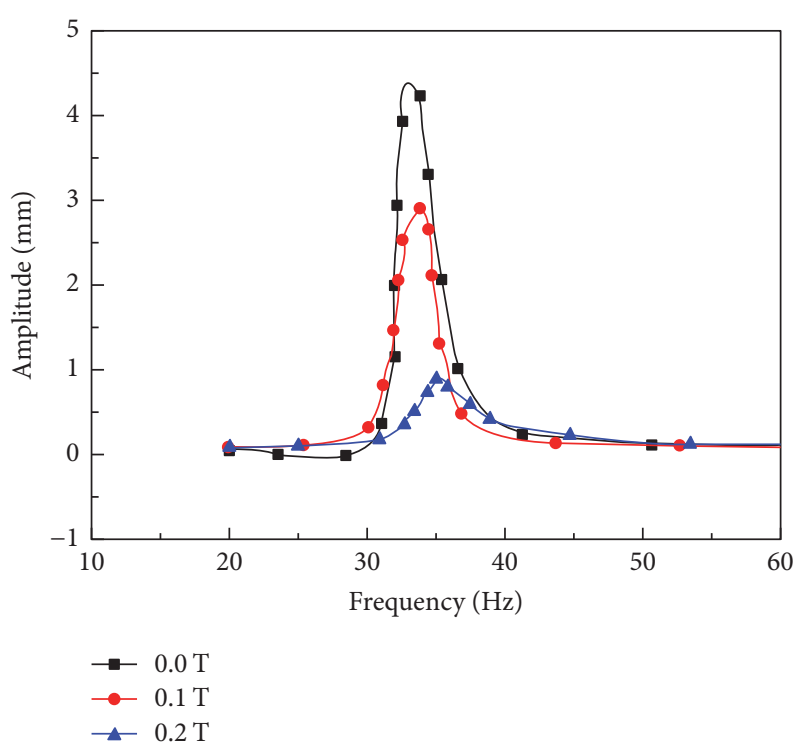

Figure 6: Amplitude versus Frequency.

advantages in vibration environment of flexible sandwich structures subjected to wide frequency spectrum of external disturbances. For example, the resonance problem under the forced vibration can be avoided by shifting the natural frequency and the settling time of the transient vibration can be reduced a lot.

It is finally remarked that optimal design of smart sandwich structures needs to be further explored to maximize the capability of the vibration parameters to the positive direction. Moreover, advanced control strategy to guarantee the structural stability and desired vibration parameters needs to be developed by integrating controllable core materials such as MR fluid.

\section{Conflicts of Interest}

The authors declare that they have no conflicts of interest.

\section{References}

[1] M. V. Gandhi, B. S. Thompson, S. B. Choi, and S. Shakir, "Electro-rheological-fluid-based articulating robotic systems," Journal of Mechanisms, Transmissions, and Automation in Design, vol. 111, no. 3, pp. 328-336, 1989.

[2] M. V. Gandhi, B. S. Thompson, and S. B. Choi, "A new generation of innovative ultra-advanced intelligent composite materials featuring electro-rheological fluids: an experimental investigation," Journal of Composite Materials, vol. 23, no. 12, pp. $1232-1255,1989$.

[3] M. V. Gandhi, B. S. Thompson, and S. B. Choi, "A proof-ofconcept experimental investigation of a slider-crank mechanism featuring a smart dynamically tunable connecting rod incorporating embedded electro-rheological fluid domains," Journal of Sound and Vibration, vol. 135, no. 3, pp. 511-515, 1989.

[4] S. B. Choi, Control of single-link flexible manipulators fabricated from advanced composite laminates and smart materials incorporating electro-rheological fluids [Ph.D. dissertation], Department of Mechanical Engineering, Michigan State University, East Lansing, Mich, USA, 1990.

[5] S. B. Choi, B. S. Thompson, and M. V. Gandhi, "An experimental investigation on smart laminated composite structures featuring embedded electro-rheological fluid domains for vibrationcontrol applications," Composites Part B: Engineering, vol. 2, no. 5-7, pp. 543-559, 1992.

[6] S.-B. Choi, Y.-K. Park, and J.-D. Kim, "Vibration characteristics of hollow cantilevered beams containing an electro-rheological fluid," International Journal of Mechanical Sciences, vol. 35, no. 9, pp. 757-768, 1993. 
[7] S.-B. Choi, B. S. Thompson, and M. V. Gandhi, "Experimental control of a single-link flexible arm incorporating electrorheological fluids," Journal of Guidance, Control, and Dynamics, vol. 18, no. 4, pp. 916-919, 1995.

[8] Y. Choi, A. F. Sprecher, and H. Conrad, "Vibration characteristics of a composite beam containing an electrorheological fluid," Journal of Intelligent Material Systems and Structures, vol. 1, no. 1, pp. 91-104, 1990.

[9] Y. Choi, A. F. Sprecher, and H. Conrad, "Response of electrorheological fluid-filled laminate composites to forced vibration," Journal of Intelligent Material Systems and Structures, vol. 3, no. 1, pp. 17-29, 1992.

[10] J. S. Leng, Y. J. Liu, S. Y. Du, L. Wang, and D. F. Wang, "Active vibration control of smart composites featuring electro-rheological fluids," Applied Composite Materials, vol. 2, no. 1, pp. 5965, 1995.

[11] M. Yalcintas and J. P. Coulter, "Analytical modeling of electrorheological material based adaptive beams," Journal of Intelligent Material Systems and Structures, vol. 6, no. 4, pp. 488-497, 1995.

[12] J.-Y. Yeh and L.-W. Chen, "Vibration of a sandwich plate with a constrained layer and electrorheological fluid core," Composite Structures, vol. 65, no. 2, pp. 251-258, 2004.

[13] J.-Y. Yeh and L.-W. Chen, "Finite element dynamic analysis of orthotropic sandwich plates with an electrorheological fluid core layer," Composite Structures, vol. 78, no. 3, pp. 368-376, 2007.

[14] M. Yalcintas and H. Dai, "Magnetorheological and electrorheological materials in adaptive structures and their performance comparison," Smart Materials and Structures, vol. 8, no. 5, pp. 560-573, 1999.

[15] M. Yalcintas and H. Dai, "Vibration suppression capabilities of magnetorheological materials based adaptive structures," Smart Materials and Structures, vol. 13, no. 1, pp. 1-11, 2004.

[16] Q. Sun, J.-X. Zhou, and L. Zhang, "An adaptive beam model and dynamic characteristics of magnetorheological materials," Journal of Sound and Vibration, vol. 261, no. 3, pp. 465-481, 2003.

[17] Z.-F. Yeh and Y.-S. Shih, "Dynamic characteristics and dynamic instability of magnetorheological material-based adaptive beams," Journal of Composite Materials, vol. 40, no. 15, pp. 1333-1359, 2006.

[18] B. Hu, D. Wang, P. Xia, and Q. Shi, "Investigation on the vibration characteristics of a sandwich beam with smart compositesMRF,' World Journal Modelling Simulation, vol. 2, pp. 201-206, 2006.

[19] R. Vasudevan, R. Sedaghati, and S. Rakheja, "Vibration analysis of a multi-layer beam containing magnetorheological fluid," Smart Materials and Structures, vol. 19, no. 1, Article ID 015013, 2010.

[20] V. Lara-Prieto, R. Parkin, M. Jackson, V. Silberschmidt, and Z. Kęsy, "Vibration characteristics of MR cantilever sandwich beams: Experimental study," Smart Materials and Structures, vol. 19, no. 1, Article ID 015005, 2010.

[21] R. Vasudevan, R. Sedaghati, and S. Rakheja, "Optimal vibration control of beams with total and partial MR-fluid treatments," Smart Materials and Structures, vol. 20, no. 11, Article ID 115016, 2011.

[22] Y. H. Li, B. Fang, F. M. Li, J. Z. Zhang, and S. Li, "Dynamic analysis of sandwich plates with a constraining layer and a magnetorheological fluid core," Polymer Composite, vol. 19, no. 4-5, pp. 295-302, 2011.
[23] J.-Y. Yeh, "Vibration analysis of sandwich rectangular plates with magnetorheological elastomer damping treatment," Smart Materials and Structures, vol. 22, no. 3, Article ID 035010, 2013.

[24] V. Rajamohan, S. Rakheja, and R. Sedaghati, "Vibration analysis of a partially treated multi-layer beam with magnetorheological fluid," Journal of Sound and Vibration, vol. 329, no. 17, pp. 34513469, 2010.

[25] V. Rajamohan, V. Sundararaman, and B. Govindarajan, "Finite element vibration analysis of a magnetorheological fluid sandwich beam," in Proceedings of the International Conference on Design and Manufacturing (IConDM '13), vol. 64, pp. 603-612, July 2013.

[26] V. Rajamohan and M. Ramamoorthy, "Dynamic characterization of non-homogeneous magnetorheological fluids based multi-layer beam," Applied Mechanics and Materials, vol. 110-116, pp. 105-112, 2012.

[27] V. Rajamohan and P. Natarajan, "Vibration analysis of a rotating magnetorheological fluid sandwich beam," in Proceedings of the International Conference on Advanced Research in Mechanical Engineering (ICARME '12), pp. 978-93, Tirupati, India, May 2012.

[28] S. Momeni, A. Zabihollah, and M. Behzad, "Experimental works on dynamic behavior of laminated composite beam incorporated with magneto-rheological fluid under random excitation," in Proceedings of the 3rd International Conference on Mechatronics and Robotics Engineering, pp. 156-161, Paris, France, Feburary 2017.

[29] C. A. Walikar, S. Kolekar, R. Hanumantharaya, and K. Raju, "A study on vibration characteristics of engine oil based magnetorheological fluid sandwich beam," Journal of Mechanical Engineering and Automation, vol. 5, no. 3, pp. 84-88, 2015.

[30] S. B. Joshi, "Vibration study of magnetorheological fluid filled sandwich beams," International Journal of Applied Research in Mechanical Engineering, vol. 2, no. 2, pp. 100-104, 2012.

[31] R. H. Scanlan, "Linear damping models and causality in vibrations," Journal of Sound and Vibration, vol. 13, no. 4, pp. 499-503, 1970.

[32] E. E. Ungar and J. Kerwin, "Loss factors of viscoelastic systems in terms of energy concepts," The Journal of the Acoustical Society of America, vol. 34, pp. 954-957, 1962.

[33] V. D. Naylor, "Some fallacies in modern damping theory," Journal of Sound and Vibration, vol. 11, no. 2, pp. 278-280, 1970. 


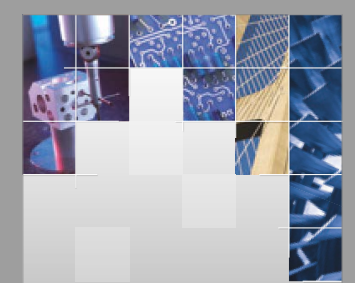

\section{Enfincering}


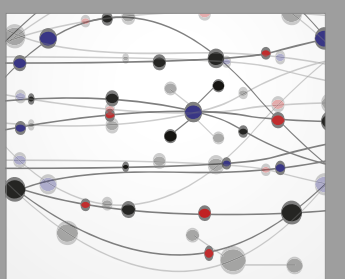

The Scientific World Journal

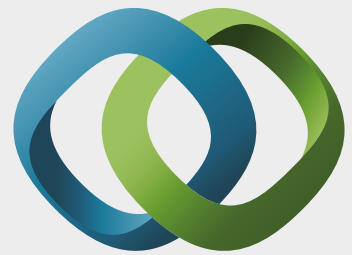

\section{Hindawi}

Submit your manuscripts at

https://www.hindawi.com
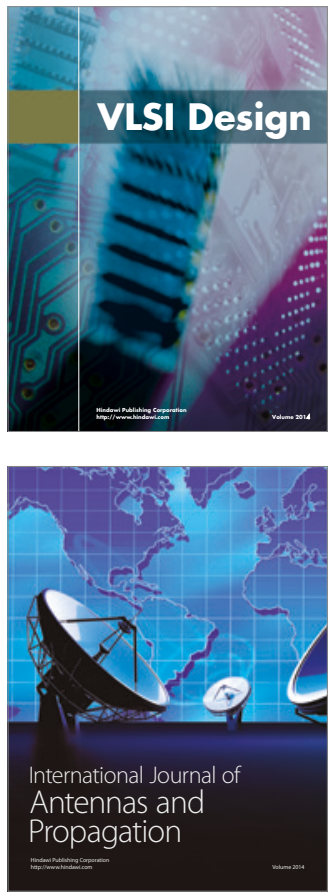

\section{Rotating}

Machinery


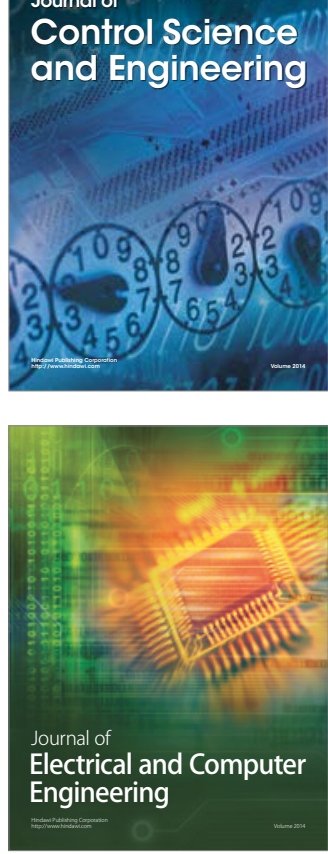

Distributed

Journal of

Control Science

and Engineering
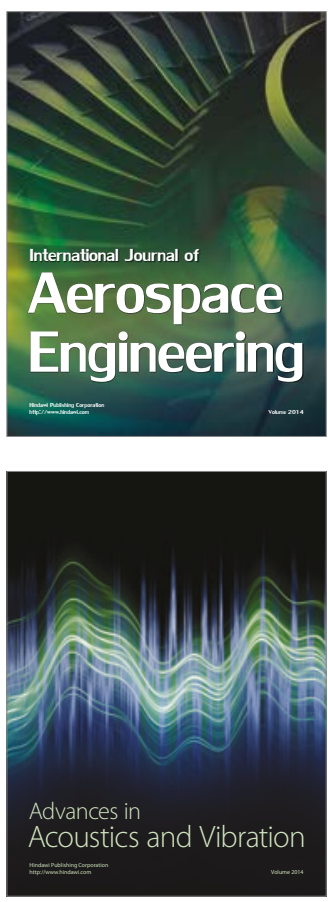

Sensor Networks 